\title{
Processo de supervisão dos enfermeiros no ambiente hospitalar e sua influência na
}

\section{qualidade assistencial}

\author{
Nurses supervision process in the hospital environment and its influence on quality care \\ Proceso de supervisión de enfermeras en el ambiente hospitalario y su influencia enla calidad \\ atención
}

Recebido: 29/07/2021 | Revisado: 07/08/2021 | Aceito: 13/08/2021 | Publicado: 15/08/2021

Tatiane Nascimento de Menezes

ORCID: https://orcid.org/0000-0002-0319-2461

Universidade Federal de Sergipe, Brasil

E-mail: ttmenezes8@hotmail.com

José Marcos de Jesus Santos

ORCID: https://orcid.org/0000-0001-5122-1469

Universidade de São Paulo, Brasil E-mail: jsmarcos@usp.br

Maria Fernanda de Sá Camarço ORCID: https://orcid.org/0000-0002-5012-2968 Universidade Tiradentes, Brasil

E-mail: mfscamarco@gmail.com

Josefa Nayara dos Santos Soares ORCID: https://orcid.org/0000-0001-5983-7161 Universidade Tiradentes, Brasil

E-mail: nayara.soaresn8@gmail.com

Maria Vitória Santos de Jesus ORCID: https://orcid.org/0000-0002-8036-9056 Universidade Tiradentes, Brasil

E-mail: vitoria_stos15@hotmail.com

Anna Letícia Ferreira Gomes

ORCID: https://orcid.org/0000-0002-1949-4544

Universidade Tiradentes, Brasil

E-mail: annaleticiafg@gmail.com

Nara Fabiana Mariano

ORCID: https://orcid.org/0000-0002-3657-4730

Universidade Estadual de Campinas, Brasil

E-mail: fale.com.nara@gmail.com

Rebecca Maria Oliveira de Góis

ORCID: https://orcid.org/0000-0002-3935-5904

Universidade Federal da Bahia, Brasil

E-mail: rebecca.gois@hotmail.com

\begin{abstract}
Resumo
Objetivo: Avaliar as evidências científicas acerca do processo de supervisão dos enfermeiros no ambiente hospitalar e sua influência na qualidade assistencial. Bem como identificar as técnicas e ferramentas utilizadas na supervisão em enfermagem. Método: Trata-se de um estudo de revisão integrativa que seguiu as seis fases preconizadas para a sua elaboração. A coleta de dados foi realizada nas seguintes bases: Education Resources Information Center, Scientific Electronic Library Online, Periódicos Coordenação de Aperfeiçoamento de Pessoal de Nível Superior/ Ministério da Educação e Biblioteca Virtual em Saúde, através dos Descritores em Ciências da Saúde e do Medical Subjetc Headings. Resultados: Foram encontrados 1059 artigos, dos quais, após serem aplicados os critérios de exclusão, foram incluídos no estudo 15 artigos. Destes, 66,66\% eram de produção internacional e 33,33\% de produção nacional, sendo 33,5\% estudos de caráter qualitativo, 6,6\% quantitativo, 6,6\% Ensaio Clínico Randomizado, 13,3\% estudo longitudinal, 6,6\% relato de experiência, 27\% estudo transversal e 6,6\% estudo teórico. Dentre os resultados foram analisadas três categorias, sendo elas: Supervisão no processo de trabalho do enfermeiro; Instrumentos de supervisão; e Estratégias inovadoras no processo de supervisão do trabalho. Conclusão: Os impactos da supervisão produzem resultados positivos na qualidade assistencial. Portanto, o enfermeiro deve aperfeiçoar seus conhecimentos científicos acerca da temática, e buscar estratégias e instrumentos que otimizem sua atuação profissional nos estabelecimentos de saúde.
\end{abstract}

Palavras-chave: Cuidados de enfermagem; Qualidade da assistência à saúde; Supervisão hospitalar. 


\begin{abstract}
Objective: Evaluate scientific evidence about the process of supervision of nurses in the hospital environment and its influence on quality of care. As well as identifying the techniques and tools used in nursing supervision. Method: This integrative review study followed the six stages recommended for its preparation. Data collection was carried out in the following databases: Education Resources Information Center, Scientific Electronic Library Online, Periodicos Coordination for the Improvement of Higher Education Personnel/Ministry of Education and Virtual Health Library, through Health Sciences Descriptors and Medical Subject Headings. Results: 1059 articles were found, of which, after applying the exclusion criteria, 15 articles were included in the study. Of these, $66.66 \%$ were of international production and $33.33 \%$ of national production, $33.5 \%$ being qualitative studies, $6.6 \%$ quantitative, 6.6\% Randomized Clinical Trial, $13.3 \%$ longitudinal study, $6.6 \%$ experience report, $27 \%$ cross-sectional study and $6.6 \%$ theoretical study. Among the results, three categories were analyzed, namely: Supervision in the nurse's work process; supervisory instruments; and Innovative strategies in the work supervision process. Conclusion: The impacts of supervision produce positive results in the quality of care. Therefore, nurses must improve their scientific knowledge on the subject, and seek strategies and instruments that optimize their professional performance in health facilities.
\end{abstract}

Keywords: Nursing care; Quality of health care; Hospital administration.

\title{
Resumen
}

Objetivo: Evaluar la evidencia científica sobre el proceso de supervisión de dos enfermeras en el ámbito hospitalario y su influencia en la calidad de la atención. Así como identificar las técnicas y herramientas utilizadas en la vigilancia de enfermedades. Método: se trata de un estudio de revisión integradora que siguió seis fases recomendadas para su elaboración. Los datos se encontraban en cola en las siguientes bases de datos: Centro de Información de Recursos Educativos, Biblioteca Electrónica Científica en Línea, Coordinación de Revistas de Educación Superior / Ministerio de Educación y Biblioteca Virtual en Salud, a través de dos Descriptores de Ciencias de la Salud y los Encabezamientos de Materia Médica. Resultados: se encontraron 1059 ítems, dos de los cuales se aplicarán a los criterios de exclusión, incluidos 15 ítems. Desde entonces, 66,66\% fueron de producción internacional y $33,33 \%$ de producción nacional, siendo 33,5\% estudios cualitativos, $6,6 \%$ cuantitativos, $6,6 \%$ ensayo clínico aleatorizado, 13,3\% estudio longitudinal, 6,6\% informe de experiencia, $27 \%$ estudio transversal y $6,6 \%$ estudio teórico. Entre los resultados analizados en tres categorías, fueron: No supervisar el proceso de trabajo de enfermería; instrumentos de supervisión; y Estrategias innovadoras y proceso de trabajo no supervisado. Conclusión: Los impactos de la supervisión producen resultados positivos en la calidad de la atención. Por tanto, el enfermero debe preparar sus conocimientos científicos sobre el tema, y buscar estrategias e instrumentos que optimicen su desempeño profesional para el establecimiento de nuestra salud.

Palabras clave: Atención de enfermería; Calidad de la atención de salud; Administración hospitalaria.

\section{Introdução}

No contexto histórico acerca da enfermagem, a necessidade de saúde e a complexidade dos serviços estão restritamente interligadas e são estudadas a todo o momento, visando às boas práticas de enfermagem frente ao cuidado ao paciente. Nota-se a necessidade de aprimoramento e reorganização do trabalho de enfermagem para permitir a mudança do paradigma do modelo gerencial obsoleto. Por isso, a qualidade da assistência prestada é a prioridade de qualquer estabelecimento de saúde, pois é um fator essencial do cuidado e uma meta de melhoria contínua que requer motivação e investimento profissional e da própria instituição (Pinto, Santos \& Pires, 2017), (Chaves et al., 2017).

Sendo assim, no século XXI, a supervisão deixa de ser apenas um instrumento de gestão com o objetivo de controle, de caráter punitivo e fiscalizador e passa a ser considerada um método de intervenção no gerenciamento em enfermagem, atuando não somente no processo de trabalho, mas também nos agentes envolvidos. Essa mudança é consequência das transformações ocorridas em nível nacional e mundial sobre a prática supervisiva dos enfermeiros e de discussões contínuas sobre as políticas públicas de gestão do cuidado em enfermagem e saúde e os impactos das práticas assistenciais e gerenciais nos processos de cuidado (Dias et al., 2018).

Após as modificações ao longo dos anos, o fazer supervisivo, atualmente, assume uma definição contemporânea que ainda considera o caráter de controle da supervisão, visto que é inerente ao processo, porém agrega a dimensão educativa, com ênfase no desenvolvimento das pessoas envolvidas no trabalho. Esse enfoque se dá pela necessidade de repensar as 
práticas gerenciais para serem pautadas nos princípios de integralidade, do agir interprofissional e, sobretudo, da satisfação dos colaboradores (Góis \& Servo, 2017).

No que se refere à execução e qualidade da supervisão, esta sofre influência de alguns fatores como: conhecimento científico inadequado pelos profissionais, não preparação dos acadêmicos desde o período da formação para os saberes gerenciais pelas instituições de ensino, supressão de autonomia e carga de trabalho excessiva (Brasil, 2018). Dada sua importância, a atividade assistencial do Técnico e Auxiliar de Enfermagem somente poderá ser realizada sob a direção/supervisão do Profissional Enfermeiro conforme estabelecido pela Lei do Exercício Profissional nº 7.498/86 (Soares, Camelo, Resck \& Terra, 2016).

Sabendo que, o mercado de trabalho exige cada vez mais dos profissionais enfermeiros, os acadêmicos de enfermagem já devem passar por uma preparação desde a graduação quanto ao desenvolvimento das competências e habilidades necessárias a prática profissional, conforme as Diretrizes Curriculares Nacionais (DCNs), descritas no Art. $4^{\circ}$ da Resolução CNE/CES no 3/2001, sendo elas: atenção à saúde, administração e gerenciamento, liderança, trabalho em equipe, tomada de decisão, comunicação, educação permanente (Dias, Toledo, Amaro \& Siman, 2017).

Destaca-se a supervisão de enfermagem, como um instrumento de suma importância para a excelência da assistência prestada pelo enfermeiro, pois ela influencia diretamente na qualidade da assistência prestada, devido aos seus meios de avaliação e organização. Sendo assim, o enfermeiro deve apropriar-se do processo supervisão e colocá-lo em prática no seu processo de trabalho. Para essa apropriação, faz-se necessário uma discussão contínua nos serviços de saúde para averiguar potencialidades e fragilidade do serviço que influencie negativamente e desqualifique o processo da supervisão em enfermagem (Carvalho, Gama \& Salimena, 2017).

Portanto, o presente trabalho foi motivado mediante inquietação observada durante o estágio curricular do componente curricular Enfermagem na Gestão Hospitalar do Curso de Enfermagem de uma Instituição de Ensino Superior do Estado de Sergipe que permitiu uma aproximação da vivência das funções gerenciais do enfermeiro e então surgiu o interesse em conhecer as estratégias e os instrumentos de supervisão pelos enfermeiros da instituição concedente do estágio, devido a sua importância na prestação de serviços de qualidade.

Este estudo justifica-se pela necessidade de aprofundamento sobre a temática abordada, já que existe uma preocupação na área da gestão hospitalar, no que se refere a garantia da qualidade dos serviços prestados ao cliente, pois, apesar da relação positiva entre a supervisão em enfermagem e a assistência de qualidade, cada profissional a executa da forma que lhe é pertinente. Resultando em improdutividade, comprometimento da segurança do paciente, maior índice de erros, desorganização e gerenciamento de recursos humanos inadequados.

\section{Metodologia}

\subsection{Tipo de estudo}

Trata-se de um estudo de revisão integrativa. Destaca-se que o estudo de revisão proposto combina "dados da literatura teórica e empírica, para fornecer uma compreensão mais abrangente de um determinado fenômeno ou problema de saúde, além de incorporar um vasto leque de propósitos: definição de conceitos, revisão de teorias e evidências, e análise de problemas metodológicos de um tópico particular, ou seja, é uma "metodologia que proporciona a síntese do conhecimento e a incorporação da aplicabilidade de resultados de estudos significativos na prática. Por se tratar de método explícito e sistemático para identificar, selecionar e avaliar a qualidade de evidências, as revisões sistemáticas são tipos de estudos produzidos por uma metodologia confiável, rigorosa e auditável (Cecílio \& Oliveira, 2017).

Segundo Silva et al. (2017), a revisão integrativa contribui para uma possível repercussão benéfica na qualidade dos cuidados prestados ao paciente. Pontua-se, então, que o impacto da utilização da revisão integrativa se dá não somente pelo 
desenvolvimento de políticas, protocolos e procedimentos, mas também no pensamento crítico que a prática diária necessita. Posto isso, para elaboração da revisão integrativa é necessário seguir seis fases distintas.

\section{Primeira fase: elaboração da pergunta norteadora}

A definição da pergunta norteadora é a fase mais importante da revisão, pois determina quais serão os estudos incluídos, os meios adotados para a identificação e as informações coletadas de cada estudo selecionado. Logo, inclui a definição dos participantes, as intervenções a serem avaliadas e os resultados a serem mensurados (Sousa, Vieira, Severino \& Antunes, 2017).

Foi utilizada a estratégia PICO (População, Intervenção, Comparação da intervenção, se aplicável, e Outcome) na formulação da questão norteadora (Figura 1). Que resultou no seguinte questionamento: Quais são as evidências científicas acerca do processo de supervisão dos enfermeiros no ambiente hospitalar e sua influência na qualidade assistencial?

Diante do exposto para melhor entendimento dessa questão, formulou-se o seguinte objetivo: Avaliar as evidências científicas acerca do processo de supervisão dos enfermeiros no âmbito hospitalar e sua influência na qualidade assistencial.

Figura 1 - Estratégia PICO Adaptada.

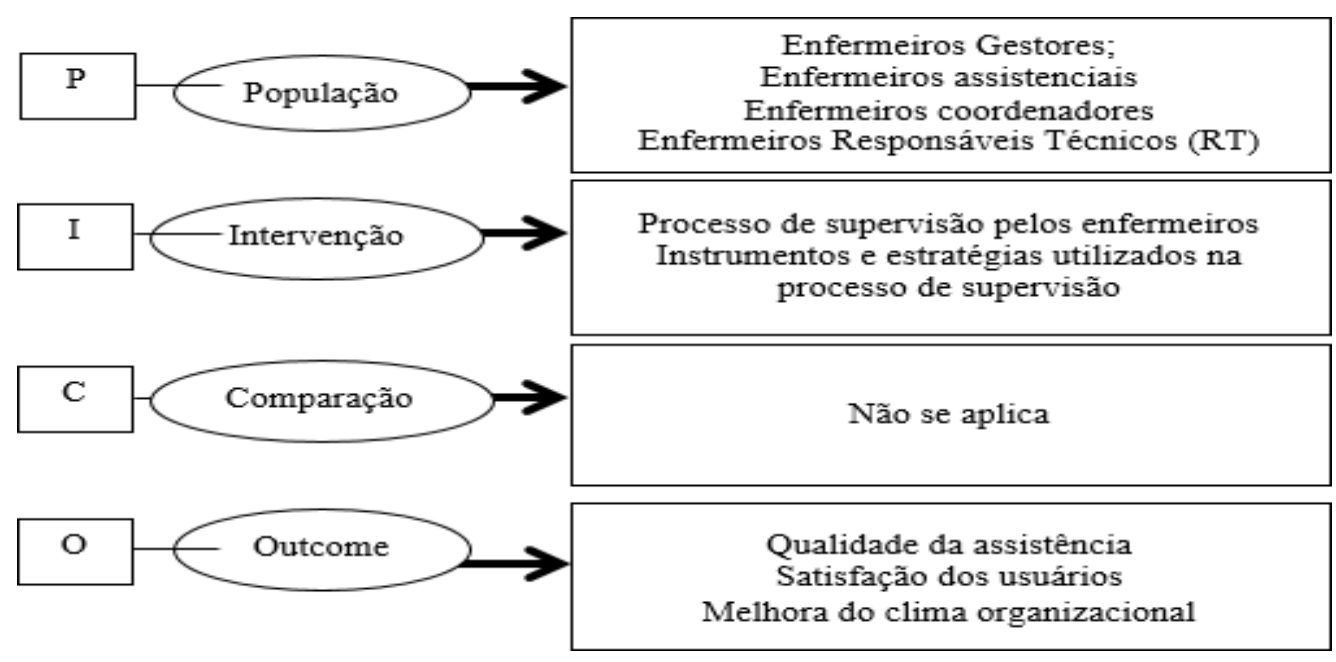

Fonte: Autores (2021).

\section{Segunda fase: busca ou amostragem na literatura}

Intrinsecamente relacionada à fase anterior, a busca em base de dados deve ser ampla e diversificada, contemplando a procura em bases eletrônicas, busca manual em periódicos, as referências descritas nos estudos selecionados, o contato com pesquisadores e a utilização de material não-publicado. Deve-se expor e discutir claramente os critérios de inclusão e exclusão de artigos devendo ser realizada em concordância com a pergunta norteadora, considerando os participantes, a intervenção e os resultados de interesse (Mendes, Silveira \& Galvão, 2019).

Utilizou-se como critérios de inclusão artigos disponíveis na íntegra nos idiomas inglês, português e espanhol, quantitativas e qualitativas, que abordassem a temática da pesquisa na área de enfermagem, no recorte temporal de 2015 a 2020. O recorte temporal escolhido justifica-se devido à evolução do conceito de supervisão ocorrida ao longo dos anos. Além disso, o ano de 2015, no contexto de supervisão de enfermagem no estado de Sergipe, é marcado por um parecer técnico $\mathrm{n}^{\circ} 17$, de 24 de abril de 2015, emitido após denúncias de procedimentos sendo realizados por técnicos, auxiliares e 
estagiários de uma determinada unidade sem a supervisão do profissional enfermeiro mesmo após determinação da Lei Federal 7.498/1986 e Decreto-lei 94.406/1987 (Coren, 2015).

Foram utilizados como critérios de exclusão: artigos publicados fora do período estabelecido, os que não respondiam à pergunta norteadora, estudos de outras áreas de conhecimento e literatura cinza como reflexões, revisões, teses e dissertações.

\section{Terceira fase: coleta de dados}

A coleta de dados foi realizada nas seguintes bases de dados e bibliotecas eletrônicas: Education Resources Information Center (ERIC), PubMed/MEDLINE, Periódicos Coordenação de Aperfeiçoamento de Pessoal de Nível Superior/ Ministério da Educação (CAPS/MEC), Scientific Electronic Library Online (SciElo) e Biblioteca Virtual em Saúde (BVS). Foram selecionados os descritores indexados nos Descritores de Saúde (DeCS), sendo eles: "Supervisão Hospitalar"; "Cuidados de Enfermagem" e "Qualidade da Asistência à Saúde", seus correspondentes na língua inglesa e espanhola, respectivamente: "Hospital Administration"; "Nursing Care"; "Quality of Health Care" e "Administración Hospitalaria" and "Atención de Enfermería" and "Calidad de La Atención de Salud".

A estratégia de busca no PubMed/MEDLINE contou com o uso do Medical Subject Headings (MeSH): "nursing care management"; "hospital administration"; "quality of health care"; "supervisory nursing". Visando compor a metodologia de busca nas plataformas supracitadas, utilizou-se os operadores booleanos: "AND", "OR" ou "NOT", sendo o AND utilizado para localizar estudos convergentes, o OR para artigos que se refere um ou outro tema, por fim o operador NOT, é utilizado para eliminar um assunto da busca (Mendes, Silveira \& Galvão, 2019).

A estratégia de busca nas bases de dados foi construída utilizando operadores booleanos para atender aos objetivos propostos do estudo e seguindo os critérios de inclusão estabelecidos para revisões integrativas, conforme explicitado na Tabela 1. 
Tabela 1 - Estratégia de busca nas bases de dados, 2021.

\begin{tabular}{|c|c|c|c|}
\hline Base de dados & Strategy & Amount & $N$ \\
\hline ERIC & $\begin{array}{l}\text { *(("Hospital Administration" AND "Nursing Care" AND } \\
\text { "Quality of Health Care")) }\end{array}$ & $\begin{array}{l}\text { Founds } \\
\text { Exclued } \\
\text { Total selected }\end{array}$ & $\begin{array}{c}14 \\
14 \\
0\end{array}$ \\
\hline SciElo & $\begin{array}{l}\text { *(("Supervisão Hospitalar" AND; "Cuidados de Enfermagem" } \\
\text { AND "Qualidade da assistência à } \\
\text { Saúde")) }\end{array}$ & $\begin{array}{c}\text { Founds } \\
\text { Exclued } \\
\text { Total selected }\end{array}$ & $\begin{array}{l}53 \\
04\end{array}$ \\
\hline $\begin{array}{l}\text { CAPS/ } \\
\text { MEC }\end{array}$ & $\begin{array}{l}\text { *(("Supervisão Hospitalar" AND; "Cuidados de Enfermagem" } \\
\text { AND "Qualidade da assistência à saúde")) }\end{array}$ & $\begin{array}{l}\text { Founds } \\
\text { Exclued } \\
\text { Total selected }\end{array}$ & $\begin{array}{c}73 \\
73 \\
0\end{array}$ \\
\hline BVS & $\begin{array}{l}\text { *(("Supervisão Hospitalar" AND; "Cuidados de Enfermagem" } \\
\text { AND 'Qualidade da assistência à saúde")) }\end{array}$ & $\begin{array}{l}\text { Founds } \\
\text { Exclued } \\
\text { Total selected }\end{array}$ & $\begin{array}{c}326 \\
322 \\
04\end{array}$ \\
\hline $\begin{array}{l}\text { PubMed/ } \\
\text { MEDLINE } \\
\text { *MeSH }\end{array}$ & 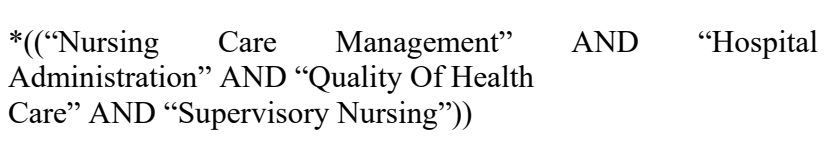 & $\begin{array}{c}\text { Founds } \\
\text { Exclued } \\
\text { Total selected }\end{array}$ & $\begin{array}{c}582 \\
07\end{array}$ \\
\hline
\end{tabular}

Fonte: Autores (2021).

\section{Quarta fase: análise crítica dos estudos incluídos}

Análoga à análise dos dados das pesquisas convencionais, esta fase demanda uma abordagem organizada para ponderar o rigor e as características de cada estudo. A Prática Baseada em Evidências focaliza, em contrapartida, sistemas de classificação de evidências caracterizados de forma hierárquica, dependendo da abordagem metodológica adotada. Para auxiliar na escolha da melhor evidência possível, propõe-se uma hierarquia das evidências, segundo o delineamento da pesquisa, que é um dos itens a serem analisados nesta fase (Galvão \& Ricarte, 2019).

O processo de seleção e avaliação dos artigos foi realizado de maneira independente. Além disso, posteriormente, realizou-se a verificação dos artigos, buscando identificar duplicidades, divergências e convergências, conforme ilustrado na Figura 2. 
Figura 2 - Fluxograma Prisma, 2021.

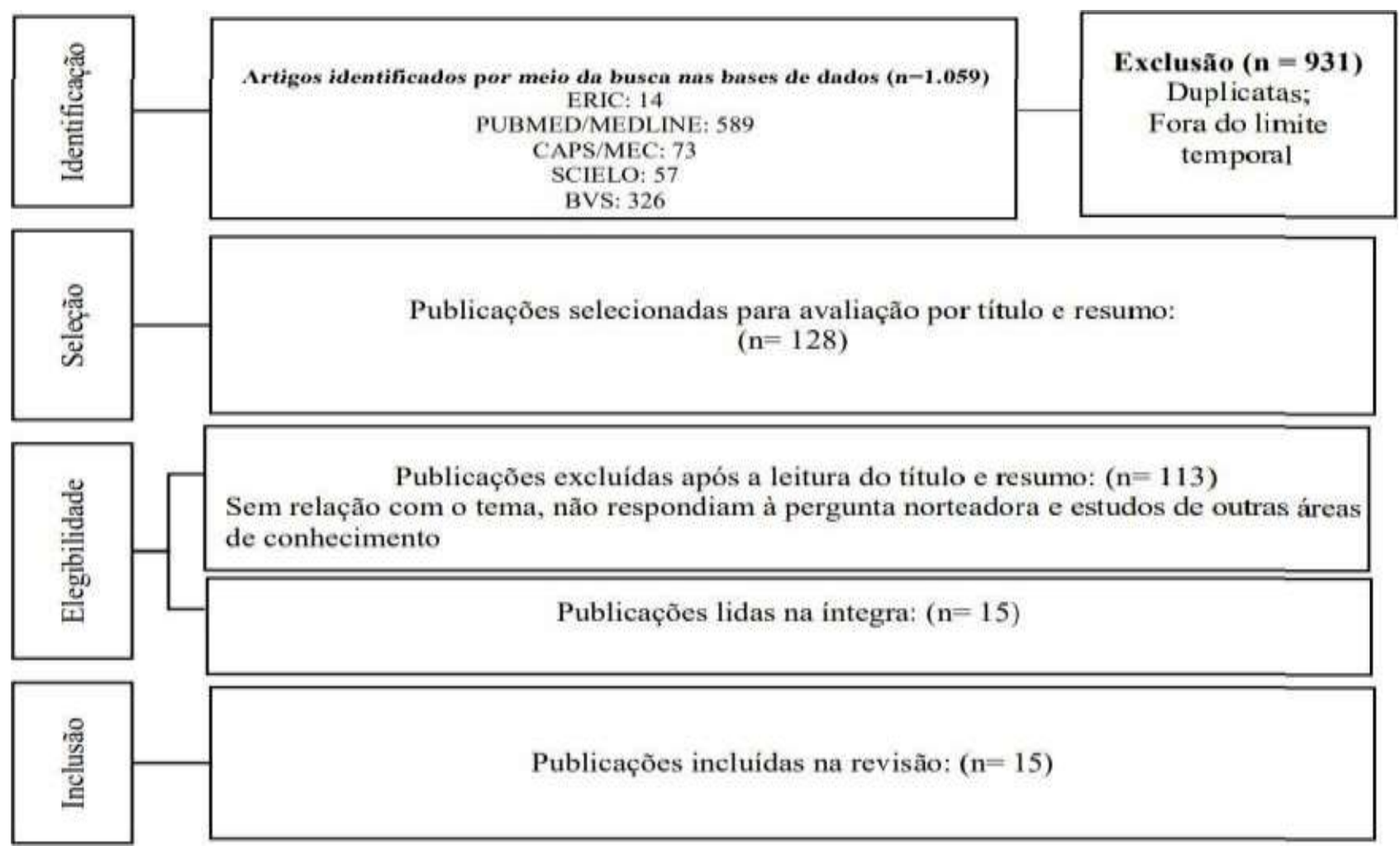

Fonte: Autores (2021).

Para avaliar a classificação das evidências de forma hierárquica utilizou-se o critério o delineamento metodológico. Nível 1: evidências resultantes da meta-análise de múltiplos estudos clínicos controlados e randomizados; Nível 2: evidências obtidas em estudos individuais com delineamento experimental; Nível 3: evidências de estudos quase-experimentais; Nível 4: evidências de estudos descritivos (não-experimentais) ou com abordagem qualitativa; Nível 5: evidências provenientes de relatos de caso ou de experiência; Nível 6: evidências baseadas em opiniões de especialistas (Silva et al., 2017).

\section{Resultados}

\section{Quinta fase: discussão dos resultados}

Nesta etapa, a partir da interpretação e síntese dos resultados, comparam-se os dados evidenciados na análise dos artigos ao referencial teórico (Galvão \& Ricarte, 2019).

Os estudos analisados podem ser caracterizados quantitativamente do seguinte modo: 9 (66,66\%) são produções internacionais e $6(33,33 \%)$ são nacionais. Quanto à natureza metodológica, $5(33,5 \%)$ possuem enfoque qualitativo, 1 (6,6\%) enfoque quantitativo, 1 (6,6\%) Ensaio Clínico Randomizado, 2 (13,3\%) estudo longitudinal, 1 (6,6\%) relato de experiência, 4 (27\%) estudo transversal e 1 (6,6\%) estudo teórico. Quanto ao local dos estudos, identificou-se que 6 das publicações são do Brasil (33,33\%), 2 Dinamarca (13,3\%), 2 Portugal $(13,3)$ e demais com 1 (6,66\%) da Austrália, Ásia, Noruega, EUA e Escócia.

Sendo assim, ao analisar as evidências científicas sobre a produção científica do período de 2015-2020 sobre o processo de supervisão dos enfermeiros no ambiente hospitalar e sua influência na qualidade assistencial verificou-se que nos artigos estudados, a grande parte dos autores são enfermeiros assistenciais, coordenadores e docentes, todavia, alguns estudosnão expõem a formação acadêmica dos autores, mas o núcleo acadêmico que estes estão inseridos, os estudos em sua 


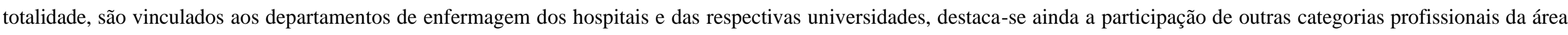
da saúde na construção dos artigos, tais como: psicologia e medicina.

Em relação a avaliação para a classificação das evidências de forma hierárquica utilizou-se o critério de delineamento metodológico, 1 (6,66\%) possuem nível 1 de evidência, 1(6,66\%) nível 3,

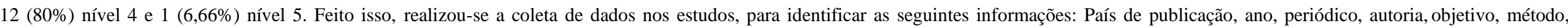
principais repercussões para enfermagem e nível de evidência. A discriminação de cada estudo utilizado nesta revisão é exposta a partir do Quadro 1 .

Quadro 1 - Distribuição das Evidências Científicas incluídas na Pesquisa. Estância, SE, Brasil, 2021

\begin{tabular}{|c|c|c|c|c|c|c|}
\hline $\begin{array}{c}\text { Base de } \\
\text { Dados }\end{array}$ & $\begin{array}{l}\text { País } \\
\text { Ano }\end{array}$ & Referência & Objetivo & Método & Repercussão para a Enfermagem & $\begin{array}{c}\text { Nível de } \\
\text { Evidência }\end{array}$ \\
\hline & $\begin{array}{c}\text { Brasil } \\
2019\end{array}$ & $\begin{array}{l}\text { Forte, E. C. N., Pires, D. E. P. } \\
\text { D., Martins, M. M. F. P. D. S., } \\
\text { Padilha, M. I. C. D. S., Ghizoni } \\
\text { Schneider, D., \& Trindade, L. D. L. } \\
\text { (2019). Processo de trabalho: } \\
\text { fundamentação para compreender os } \\
\text { erros de enfermagem. Revista da } \\
\text { Escola de Enfermagem da USP, 53. }\end{array}$ & $\begin{array}{l}\text { Identificar causas relacionadas ao } \\
\text { processo de trabalho associadas a } \\
\text { erros de enfermagem relatados em } \\
\text { jornais. }\end{array}$ & $\begin{array}{l}\text { Estudo documental e qualitativo, que } \\
\text { examinou } 112 \text { artigos publicados entre } \\
2012 \text { e } 2016 \text { em } 21 \text { jornais brasileiros de } \\
\text { alta circulação, organizados e } \\
\text { codificados em software. }\end{array}$ & $\begin{array}{l}\text { A análise identificou os resultados } \\
\text { negativos do trabalho de enfermagem, além } \\
\text { de identificar os elementos do processo de } \\
\text { trabalho que influenciaram esses } \\
\text { resultados. Os achados enfatizam a } \\
\text { importância de compreender esses erros } \\
\text { para que possam ser evitados e de revisaras } \\
\text { condições de trabalho de enfermagem para } \\
\text { garantir qualidade e segurança dos } \\
\text { cuidados. }\end{array}$ & 4 \\
\hline & $\begin{array}{c}\text { Brasil } \\
2015\end{array}$ & $\begin{array}{l}\text { Santos, J. L. G. D., \& Erdmann, A. } \\
\text { L. (2015). Governance of professional } \\
\text { nursing practice in a hospital setting: } \\
\text { a mixed methods study1. Revista } \\
\text { latino- americana de } \\
\text { enfermagem, 23, 1024-1032. }\end{array}$ & $\begin{array}{l}\text { Elaborar um modelointerpretativo } \\
\text { sobre a governança da prática } \\
\text { profissional do enfermeiro no } \\
\text { ambiente hospitalar. }\end{array}$ & $\begin{array}{l}\text { Estudo com estratégia de triangulação } \\
\text { de dados de um estudo transversal com } \\
106 \text { enfermeiros e uma Teoria } \\
\text { Fundamentada nos Dados com } 63 \\
\text { participantes. Os dados quantitativos } \\
\text { foram coletados por meio do Brazilian } \\
\text { Nursing Work Index Revised e } \\
\text { submetidos a análise estatística } \\
\text { descritiva. }\end{array}$ & $\begin{array}{l}\text { Importância da reorganização dasestruturas } \\
\text { e dos processos de governança profissional } \\
\text { de enfermagem, principalmente quanto ao } \\
\text { suporte que é fornecido para a prática } \\
\text { gerencial dos enfermeiros. }\end{array}$ & 4 \\
\hline
\end{tabular}


Research, Society and Development, v. 10, n. 10, e465101018875, 2021

(CC BY 4.0) | ISSN 2525-3409 | DOI: http://dx.doi.org/10.33448/rsd-v10i10.18875

\begin{tabular}{|c|c|c|c|c|c|c|}
\hline & $\begin{array}{l}\text { Brasil } \\
2019\end{array}$ & 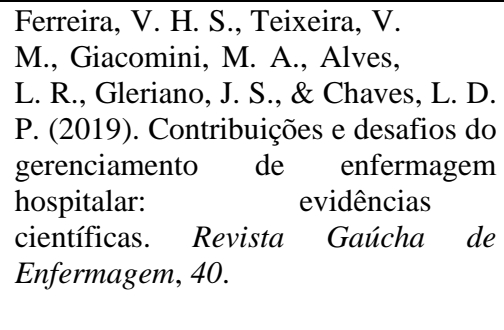 & $\begin{array}{l}\text { Analisar as evidências } \\
\text { científicas, nacionais e } \\
\text { internacionais, sobrecontribuições } \\
\text { e desafios do gerenciamento de } \\
\text { enfermagem na atenção } \\
\text { hospitalar. }\end{array}$ & $\begin{array}{l}\text { Revisão integrativa da literatura, com } \\
\text { questão norteadora: Quais evidências } \\
\text { científicas sobre o gerenciamento de } \\
\text { enfermagem na atenção hospitalar? } \\
\text { Dados coletados nas bases de dados } \\
\text { LILACS, PubMed, Scopus, } \\
\text { CINAHL e EMBASE. }\end{array}$ & $\begin{array}{l}\text { As contribuições referem-se a aspectos } \\
\text { que facilitam o desenvolvimento e } \\
\text { organização do trabalho na perspectiva } \\
\text { técnico-política, ao qualificar os processos } \\
\text { produtivos. Os desafios estão atrelados à } \\
\text { formação profissional, à satisfação com o } \\
\text { trabalho, à sobrecarga, à manutenção da } \\
\text { qualidade dos serviços, resolução de } \\
\text { conflitos e trabalho em equipe }\end{array}$ & 4 \\
\hline \multirow{3}{*}{$\begin{array}{l}\text { PubMed/ } \\
\text { MEDLIN } \\
\text { E }\end{array}$} & $\begin{array}{l}\text { Escócia } \\
2016\end{array}$ & $\begin{array}{l}\text { Rankin, J., McGuire, C., Matthews, } \\
\text { L., Russell, M., Ray, D., \& Leading } \\
\text { Better Care Research and Evaluation } \\
\text { group. (2016). Facilitators and } \\
\text { barriers to the increased supervisory } \\
\text { role of senior charge nurses: a } \\
\text { qualitative study. Journal of Nursing } \\
\text { Management, 24(3), 366-375. }\end{array}$ & $\begin{array}{l}\text { Explorar as experiências de } \\
\text { enfermeiras encarregadas de cargo } \\
\text { sênior com 'aumento de horas de } \\
\text { supervisão'. }\end{array}$ & Estudo qualitativo & $\begin{array}{l}\text { A preparação e o suporte são essenciais } \\
\text { para que os enfermeiros encarregados } \\
\text { sênior forneçam liderança clínica } \\
\text { aprimorada por meio de maior tempo de } \\
\text { supervisão. }\end{array}$ & 4 \\
\hline & $\begin{array}{c}\text { Dinamarca } \\
2018\end{array}$ & $\begin{array}{l}\text { Buus, N., Delgado, C.,Traynor, M., \& } \\
\text { Gonge, H. (2018). Resistance to } \\
\text { group clinical supervision: A } \\
\text { semistructured interview study of } \\
\text { non-participating mental health } \\
\text { nursing staffmembers. International } \\
\text { journal of mental healthnursing, } \\
27(2), 783-793 .\end{array}$ & $\begin{array}{l}\text { Examinar a resistência à } \\
\text { supervisão clínica em grupo, } \\
\text { entrevistando enfermeiros que não } \\
\text { participaram dasupervisão. }\end{array}$ & Entrevistas individuais,semiestruturadas & $\begin{array}{l}\text { Os provedores organizacionais de } \\
\text { supervisão clínica devem refletir sobre a } \\
\text { organização da supervisão clínica de } \\
\text { maneira a possibilitar a participação de } \\
\text { todos os funcionários de enfermagem e } \\
\text { sobre combinar sistematicamente e e } \\
\text { efetivamente com os membros da equipe, } \\
\text { para que o desenvolvimento profissional de } \\
\text { todos os enfermeiros seja efetivo. }\end{array}$ & 4 \\
\hline & $\begin{array}{l}\text { Austrália } \\
2015\end{array}$ & $\begin{array}{l}\text { Johansson, D. (2015). Nursing } \\
\text { clinical supervision project in a } \\
\text { Neonatal Intensive Care and a Special } \\
\text { Care Baby Unit: a best practice } \\
\text { implementation project. JBI Evidence } \\
\text { Synthesis, 13(3), 247-257. }\end{array}$ & $\begin{array}{l}\text { Conduzir uma auditoria do } \\
\text { conhecimento dos enfermeiros de } \\
\text { supervisão clínica e investigar se } \\
\text { os enfermeiros estavam cientes ou } \\
\text { se envolveram em quaisquer } \\
\text { atividades de supervisão clínica e } \\
\text { se há registro da supervisão } \\
\text { feita. }\end{array}$ & $\begin{array}{l}\text { Coleta de dados em uma amostra de } 37 \\
\text { enfermeiros. }\end{array}$ & $\begin{array}{l}\text { As unidades devem ser empreendidas } \\
\text { com estratégias para avaliar a eficácia da } \\
\text { supervisão clínica e os aspectos positivos } \\
\text { que foram relatados na literatura. }\end{array}$ & 4 \\
\hline
\end{tabular}


Research, Society and Development, v. 10, n. 10, e465101018875, 2021

\begin{tabular}{|c|c|c|c|c|c|}
\hline $\begin{array}{l}\text { EUA } \\
2016\end{array}$ & $\begin{array}{l}\text { Gonge, H., \& Buus, N. (2016). } \\
\text { Exploring organizational barriers to } \\
\text { strengthening clinical supervision of } \\
\text { psychiatric nursing staff: a } \\
\text { longitudinal controlled intervention } \\
\text { study. Issues in mental health } \\
\text { nursing, 37(5), 332-343. }\end{array}$ & $\begin{array}{l}\text { Testar se a intervenção poderia } \\
\text { aumentar a participação da } \\
\text { supervisão clínica e a eficácia das } \\
\text { práticas de supervisão existentes } \\
\text { além de explorar as restrições } \\
\text { organizacionais à implementação } \\
\text { dessas práticas. }\end{array}$ & $\begin{array}{l}\text { Estudo com desenho longitudinal } \\
\text { controlado com uma amostra dividida } \\
\text { em grupos de intervenção e controle. }\end{array}$ & $\begin{array}{l}\text { A implementação da supervisão clínica } \\
\text { deve, desde o início, contar com total apoio } \\
\text { organizacional, pois as restrições } \\
\text { organizacionais poderá obstruir a } \\
\text { implementação. Conclui-se ainda que o } \\
\text { apoio organizacional é um imperativo para } \\
\text { a implementação da supervisãoclínica. }\end{array}$ & 3 \\
\hline $\begin{array}{c}\text { Dinamarca } \\
2015\end{array}$ & $\begin{array}{l}\text { Gonge, H., \& Buus, N. (2015). Is it } \\
\text { possible to strengthen psychiatric } \\
\text { nursing staff's clinical supervision? } \\
\text { RCT of a meta-supervision } \\
\text { intervention. Journal of Advanced } \\
\text { Nursing, 71(4), 909-921. }\end{array}$ & $\begin{array}{l}\text { Testar os efeitos de uma } \\
\text { intervenção de meta-supervisão } \\
\text { em termos de participação, } \\
\text { eficácia e benefícios dasupervisão } \\
\text { clínica da equipe de enfermagem } \\
\text { psiquiátrica. }\end{array}$ & Ensaio clínico randomizado & $\begin{array}{l}\text { A intervenção teve um efeito positivo em } \\
\text { indivíduos ou enfermarias que já } \\
\text { participam ativamente da supervisão } \\
\text { clínica, o que sugeriu que indivíduos e } \\
\text { enfermarias sem práticas de supervisão } \\
\text { bem estabelecidas podem exigir } \\
\text { intervenções mais abrangentes visando } \\
\text { barreiras individuais e organizacionais à } \\
\text { supervisão clínica. }\end{array}$ & 1 \\
\hline $\begin{array}{c}\text { Noruega } \\
2019\end{array}$ & $\begin{array}{l}\text { Husebo, S. E., \& Olsen, O. E. } \\
\text { (2019). Actual clinicalleadership: a } \\
\text { shadowing studyof charge nurses } \\
\text { and doctorson-call in the emergency } \\
\text { department. Scandinavian journal of } \\
\text { trauma,resuscitation and emergency } \\
\text { medicine, 27(1), 1-9. }\end{array}$ & $\begin{array}{l}\text { Explorar as atividades realizadas } \\
\text { por líderes clínicos e identificar } \\
\text { semelhanças e diferenças entre as } \\
\text { atividades realizadas por } \\
\text { enfermeiras encarregadas e } \\
\text { aquelas realizadas por médicos de } \\
\text { plantão no Departamento de } \\
\text { Emergência após a conclusão de } \\
\text { um curso de Liderança Clínica }\end{array}$ & $\begin{array}{l}\text { Um projeto exploratório qualitativo foi } \\
\text { escolhido usando sombreamentoe } \\
\text { conversas curtas. }\end{array}$ & $\begin{array}{l}\text { A análise revelou sete eixos temáticos: } \\
\text { panorama geral de forma supervisionada da } \\
\text { equipe e dos pacientes, para planejar o } \\
\text { plantão; garantir recursos; monitorar e } \\
\text { garantir o fluxo adequado do paciente; } \\
\text { monitorar e proteger o fluxo de } \\
\text { informações; garantir atendimento e } \\
\text { tratamento ao paciente; assegurar a } \\
\text { qualidade do diagnóstico e tratamento do } \\
\text { paciente; e garantir a priorização dos } \\
\text { pacientes. }\end{array}$ & 4 \\
\hline $\begin{array}{l}\text { Asia } \\
2020\end{array}$ & $\begin{array}{l}\text { Nasurdin, A. M., Tan, C. L., \& } \\
\text { Khan, S. N. (2020). The Impact of } \\
\text { Social Support onNurses' Motivation: } \\
\text { A Cross- Sectional Study inMalaysia. } \\
\text { Asia Pacific Journal of PublicHealth, } \\
\text { 32(8), 430-435. }\end{array}$ & $\begin{array}{l}\text { Explorar } 3 \text { formas de suporte } \\
\text { social (suporte organizacional } \\
\text { percebido, suporte de supervisão } \\
\text { percebido e suporte de pares } \\
\text { percebido) na motivação dos } \\
\text { enfermeiros. }\end{array}$ & $\begin{array}{l}\text { Os dados transversais foram coletados } \\
\text { por meio de questionários de uma } \\
\text { amostra de } 354 \text { enfermeiras que } \\
\text { trabalham em hospitais públicos da } \\
\text { Malásia. O modelo hipotético foi testado } \\
\text { usando o método de mínimos quadrados } \\
\text { parciais. }\end{array}$ & $\begin{array}{l}\text { Os resultados revelaram que todas as } \\
\text { formas de apoio social, seja ele mediante } \\
\text { ao suporte organizado percebido, suportede } \\
\text { supervisão percebido e suporte de pares } \\
\text { percebido têm efeitos positivos na } \\
\text { motivação. }\end{array}$ & 4 \\
\hline
\end{tabular}


Research, Society and Development, v. 10, n. 10, e465101018875, 2021

(CC BY 4.0) | ISSN 2525-3409 | DOI: http://dx.doi.org/10.33448/rsd-v10i10.18875

\begin{tabular}{|c|c|c|c|c|c|c|}
\hline \multirow[t]{4}{*}{ BVS } & $\begin{array}{l}\text { Brasil } \\
\\
2018\end{array}$ & $\begin{array}{l}\text { Dias, C. A., Santos, D. C., de } \\
\text { Oliveira Matias, L., Servo, M. L. S., } \\
\text { de Santana, C. L. A., \& Tanaka, L. H. } \\
\text { (2018). Representações de supervisão } \\
\text { na perspectiva dos enfermeiros } \\
\text { coordenadores de um hospital de } \\
\text { ensino. Revista Baiana de } \\
\text { Enfermagem } 32, .\end{array}$ & 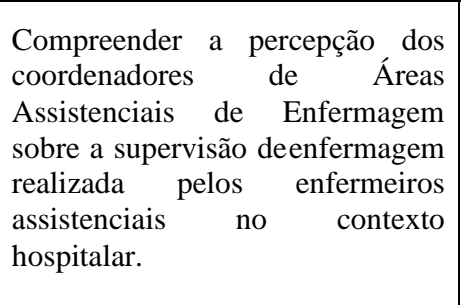 & $\begin{array}{l}\text { Estudo de abordagem qualitativa, } \\
\text { Fundamentado na Teoria das } \\
\text { Representações Sociais }\end{array}$ & 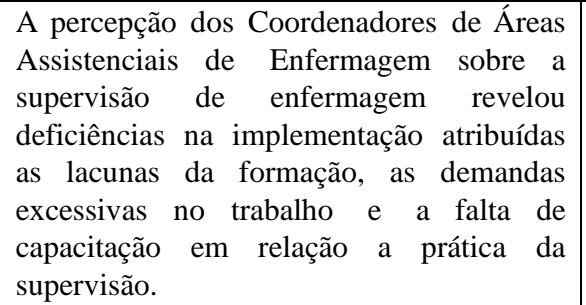 & 4 \\
\hline & $\begin{array}{l}\text { Portugal } \\
2017\end{array}$ & $\begin{array}{l}\text { Pinto, D. J. E., Santos, M. R., \& } \\
\text { Pires, R. (2017). Relevance of } \\
\text { indicators of clinical supervision } \\
\text { strategies in nursing. Revista da Rede } \\
\text { de Enfermagem doNordeste, 18(1), } \\
19 .\end{array}$ & $\begin{array}{l}\text { Identificar os indicadores de } \\
\text { estratégias de supervisão clínica } \\
\text { em enfermagem que r os } \\
\text { enfermeiros consideram mais } \\
\text { relevantes. }\end{array}$ & $\begin{array}{l}\text { Pesquisa descritiva e exploratória com } \\
\text { abordagem quantitativa }\end{array}$ & $\begin{array}{l}\text { Os indicadores identificados no estudo } \\
\text { constituem um importante passo para a } \\
\text { estruturação e avaliação dos processos de } \\
\text { supervisão, concorrendo para a melhoria da } \\
\text { qualidade e segurança dos cuidados. }\end{array}$ & 4 \\
\hline & $\begin{array}{c}\text { Portugual } \\
2020\end{array}$ & $\begin{array}{l}\text { Barroso, C., Carvalho, A. L., } \\
\text { Augusto, C., Teixeira, L. O., Teixeira, } \\
\text { A., \& Rocha, I. (2020). } \\
\text { Implementação de um modelo de } \\
\text { supervisão clínica em enfermagem- } \\
\text { modelo SAFECARE. Revista ROL de } \\
\text { Enfermería, 43(1), 50-58. }\end{array}$ & $\begin{array}{l}\text { Identificar quais são oscontributos } \\
\text { da implementação do modelo } \\
\text { contextualizado de supervisão } \\
\text { clínicadeenfermagem, Safecare no } \\
\text { contexto de cuidado da cirurgia, } \\
\text { medicina e psiquiatria e cirurgia } \\
\text { de ambulatório. }\end{array}$ & $\begin{array}{l}\text { Estudo exploratório, descritivo } \\
\text { elongitudinal. }\end{array}$ & $\begin{array}{l}\text { O modelo de supervisão clínica } \\
\text { deenfermagem com base nas necessidades } \\
\text { dos enfermeiros, o modelo SafeCare, tem } \\
\text { contribuiu para a obtenção de atendimento } \\
\text { de maior qualidade esegurança aos clientes. }\end{array}$ & 4 \\
\hline & $\begin{array}{l}\text { Uruguai } \\
2020\end{array}$ & 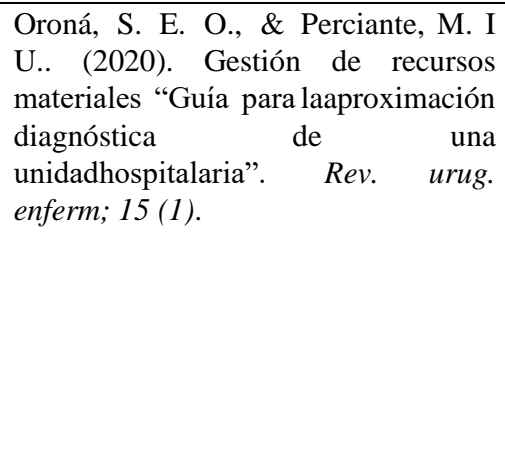 & $\begin{array}{l}\text { Identificar os pontos fortes e } \\
\text { fracos do Serviço em que é } \\
\text { aplicado e ser capaz } \\
\text { desenvolver um projeto de } \\
\text { melhoria da qualidade }\end{array}$ & $\begin{array}{l}\text { Trata-se de um estudo diagnóstico, } \\
\text { com aplicação de um Guia de } \\
\text { prática clínica, com abordagem } \\
\text { qualitativa. }\end{array}$ & $\begin{array}{l}\text { Descreveu-se cada etapa do guia junto ao } \\
\text { sistema, e como será realizado e de que } \\
\text { modo contribui e facilita o cuidado ao } \\
\text { usuário. A transformação de dados em } \\
\text { informação é uma poderosa ferramenta de } \\
\text { trabalho, estabelecer um método para o } \\
\text { conhecimento da realidade tentando fazer } \\
\text { uma captura instantânea, sendo o ponto de } \\
\text { partida para identificar e conhecer os } \\
\text { processos, medi-los e assim poder melhorá- } \\
\text { los com nuancesaspirações de excelência. }\end{array}$ & 4 \\
\hline
\end{tabular}

Fonte: Autores (2021). 


\section{Discussão}

\section{Sexta fase: apresentação da revisão integrativa}

Após leitura detalhada e contribuições de cada estudo, os resultados foram unificados e categorizados conforme semelhança de conteúdo e intervenções, e se apresentam a partir das seguintes categorias: Supervisão no Processo de Trabalho do Enfermeiro, Instrumentos de Supervisão e Estratégias Inovadoras no Processo de Supervisão de Enfermagem.

Categoria 1: Supervisão no processo de trabalho do enfermeiro. Nesta categoria estão agrupados os artigos que discutem os principais fatores relacionados à supervisão no processo de trabalho do enfermeiro por entender que o mesmo está relacionado à qualidade assistencial.

Nesse sentido, a forma como se é desenvolvido ou realizado as atividades profissionais, é chamado como processo de trabalho. O autor Forte et al. (2019), conceitua o trabalho como sendo um conjunto de procedimentos pelo qual o ser humano atua por meio da utilização de objetos ou modificação de algo para gerar algum tipo de produto de utilidade. Para ele, essa conceituação é importante, pois é durante este processo, que se torna possível a identificação de três elementos principais: a finalidade, o objeto e o instrumento, que seria respectivamente, o porquê do trabalho, o meio ou objeto que passará por transformação, e o meio que será utilizado para auxiliar na transformação.

Nessa mesma perspectiva, o estudo de Gonge e Buus (2016), abordam um pouco do que é o trabalho. No entanto, estes autores abordam uma expectativa mais voltada para o âmbito de saúde, afim de evidenciar os principais pontos. Para eles o processo de trabalho na saúde é entendido de uma maneira especifica, uma vez que o produto do trabalho não é material ou industrial, mas sim, resultante de uma assistência de saúde qualificada que é produzida e consumida concomitantemente. Desta forma, a enfermagem constitui uma categoria a qual precisa ser compreendida como um trabalho especifico, de importância social, para que os resultados de suas atividades sejam entendidos na sua total complexidade.

Seguindo o pensamento de Forte et al. (2019), o contexto de gestão em enfermagem no Brasil, é compreendida comouma ferramenta administrativa capaz de proporcionar direção no trabalho dos profissionais, gestão do cuidado, como tambémdireção na gestão dos recursos humanos e materiais. Além disso, em seu estudo, os autores ainda relatam que essa visão só ganhou conhecimento, após os adventos da política para a qualidade dos serviços prestados na saúde e da busca pela segurança do paciente.

Em contrapartida, os autores Dias et al. (2018) acreditam que para ocorrer a realização desse produto é necessário que ocorra mais que só o conhecimento dos conceitos e contextos. Segundo eles, para que haja o produto, é indispensável a utilização de instrumentos de gestão que envolva o trabalho prestado pela equipe. Dessa forma, os autores deixam subentender que a supervisão de enfermagem é uma forma de instrumento, que embasada no planejamento, implementação e avaliação do trabalho da equipe, se caracteriza como um meio de gerenciamento, o qual visa qualificar a sistematização da assistência de enfermagem em todos os níveis de atenção à saúde, qualificando o cuidado entre usuário, família e profissional.

Neste mesmo pensamento, o autor Johansson (2015), reafirma que a enfermagem é uma forma de instrumento, e que ao ser aplicada, traz alguns benefícios para a equipe, instituição, paciente e profissional. Alguns destes benefícios potenciais da supervisão são: aumento no número de enfermeiros conscientes de si mesmos e de sua enfermaria, maior conscientização desses profissionais sobre o modo de pensar, melhora a tomada de decisões e desempenho, assim capacitando-os a lidar com situações difíceis, bem como abordagem mais equilibrada da vida profissional, melhoria da satisfação e eficiência no trabalho, crescimento pessoal, aumento da autoestima e melhores relacionamentos com os gerentes. 
O Parecer $n^{\circ} 02$ do Cofen (2018), relatou a existência de diversas dificuldades que interfere na execução da supervisão pelo enfermeiro, como a falta de conhecimento dos profissionais, deficiência no preparo das instituições acadêmicas para função gerencial, falta de autonomia e sobrecarga de trabalho.

Segundo Rankin et al. (2016) e Buus, Delgado, Traynor e Gonge (2018) estes fatores acabam favorecendo com que o instrumento saia do seu foco inicial e provoque em alguns profissionais de dentro da equipe ansiedade ou ambivalência em participar da supervisão. Estes autores ainda abordam que essas reações acabam sendo comuns, uma vez que a liderança passa a não exercer o papel adequadamente, não provocando na equipe a capacitação profissional e fazendo com que o instrumento passe a ser somente um meio de verificação de tarefas cumpridas.

No entanto, os autores Dias et al. (2018), ressaltam que a partir do século XXI, a supervisão de enfermagem modificou-se e adquiriu uma perspectiva mais dialoga e participativa. Em seu estudo, os autores abordam que essa nova perspectiva, de certa forma representa um único objetivo intermeado por ações e reflexões contínuas, proporcionando aos profissionais da equipe que dela participem, uma constante reflexão na ação que venham a desenvolver.

Para eles, ao adquirir esse caráter, a supervisão de enfermagem torna-se capaz de transformar tanto os processos de trabalho, como os agentes neles envolvidos, assim evidenciando que o papel de liderança é reconhecido como habilidade vitalpara que o serviço prestado pela equipe seja eficaz, proporcionando ao paciente e a família cuidados de saúde de alta qualidade.Reafirmando essa perspectiva, os autores Ferreira et al. (2019), traz em sua pesquisa evidências cientificas sobre a importância da supervisão no processo de gerenciamento. Em seu estudo, o autor relata os desafios e as contribuições que ogerenciamento traz para o serviço, e que nesse ponto a supervisão e a coordenação da equipe ganham ênfase nas atividades desempenhadas pelo enfermeiro, pois, é exatamente na execução dessas funções que evidencia que a efetividade do serviço está vinculada à organização da unidade, ao planejamento das atividades e a educação permanente nos setores de assistência.

Categoria 2: Instrumentos de supervisão. Nesta categoria estão agrupados os artigos que discutem os principais fatores relacionados a instrumentos de supervisão por entender que o mesmo influencia diretamente na prática supervisionada do enfermeiro em seu processo de trabalho.

Santos e Erdmann (2015), defendem que os modelos de gestão são determinados por formas organizacionais, os quais possuem características de poder, autonomia, comunicação e melhora na tomada de decisão. Dentre os modelos de gestão, encontra-se o instrumento de supervisão do processo de enfermagem, o qual é composto por diversas ferramentas que direcionam e organizam a equipe de enfermagem. Sendo esse modelo uma função privativa do enfermeiro uma vez que o mesmo é responsável por planejar, organizar, coordenar, executar e avaliar os serviços de assistência de enfermagem, logo, realizar a supervisão é um papel inerente desse profissional e tem que ser exercido de forma particular.

Anteriormente, após defender que a enfermagem é uma forma de instrumento, o autor Johansson (2015), acrescenta em seu estudo que para trabalhar com o modelo de supervisão, o profissional de enfermagem tem que utilizar algumas ferramentas para melhor gerenciar a equipe. Entre essas ferramentas, o autor aborda que é possível encontrar dados estatísticos, relatórios de serviços, fichas dos usuários, para que possa ser realizada a avaliação e acompanhamento, manuais, rotinas, mapas e roteiros para melhor direcionar o profissional diante de uma determinam da situação.

Além dessas ferramentas, também é necessário que o profissional possua domínio de habilidades técnicas, as quais serão utilizadas durante a observação, orientação e avaliação das técnicas de assistência, assim como habilidades administrativas, as quais serão expostas durante o planejamento, organização, coordenação, direção e controle da equipe do serviço prestado, facilitando o cumprimento dos objetivos da supervisão pelo profissional de enfermagem (Cofen, 2018). 
Reafirmando esta ideia, os autores Pinto, Santos \& Pires (2017), abordam a existência de indicadores que ajudam avaliar o desempenho profissional de enfermagem, servindo como um facilitador na supervisão clínica, o qual possibilita o planejamento, avaliação e reorganização do processo assistencial. Além disto, estes autores descrevem que os indicadores são utilizados como diretrizes para o monitoramento, avaliação e promoção da qualidade, revelando uma situação de saúde. Dessa forma, no estudo, evidenciam que os indicadores representam uma ferramenta essencial para a operacionalização de qualquer estratégia ou programa de saúde.

Em uma linha diferenciada, os autores Kahl, Meirelles, Lanzoni, Koerich, e Cunha (2018), ressaltam que um dos meios utilizados para a avaliação é justamente as diretrizes de prática clínica em enfermagem (GPC-E), que constituem ferramentas que possibilitam exemplificar o conhecimento, as estratégias de implementação da investigação e desenvolvimento. Assim para eles, além de aperfeiçoar esse conhecimento, servem também como complemento do sistema de ajuste de riscos (SAR), que permite o reforço da eficácia e a racionalização dos custos de cuidado de saúde.

Trazendo outra sugestão, o estudo de Nasurdin, Tan e Khan (2020), abordou sobre três pontos que favorecem a melhora na assistência e desempenho geral do colaborador de enfermagem, e entre eles, um está relacionado a supervisão de enfermagem, ou seja, descreve sobre a motivação do supervisor para com sua equipe em relação ao desempenho de suas atividades.

Neste contexto, os autores dentre as três afirmações, retratam a supervisão como um instrumento motivacional, de modo que os colaboradores recebam informação e feedback, referente a prática assistencial e/ou apoio emocional. Desse modo, o estudo consegue ao final dos seus resultados afirmar o impacto positivo da supervisão da enfermagem de cunho motivacionalcomo instrumento de motivação para sua equipe, assim, favorecendo uma satisfação e assistência de qualidade.

Categoria 3: Estratégias inovadoras no processo de supervisão de enfermagem. Nesta categoria estão agrupados os artigos que discutem os principais fatores relacionadas as estratégias inovadoras no processo de supervisão de enfermagem, visto que as mesmas permitem um avanço e inovação frente ao processo de supervisão.

Segundo os autores Gonge e Buss (2016), durante a realização do plano gerencial, encontra-se a prática de supervisão que integra gerentes planejadores e pessoas operacionais, além de envolver outros profissionais que agem de forma direta e indireta na assistência. Assim, os autores abordam que essa estratégia tem como objetivo orientá-los a fim de cumprir o que foi planejado durante aquele tempo, administrando as demandas dos pacientes e de gastos com materiais, além da resolução ou amenização de tensões e conflitos entre as equipes de trabalho e outras questões cotidianas.

De forma geral, mais uma vez o pensamento do estudo de Johansson (2015), é abordado, dessa vez ressaltando a vertente que o contexto no qual o profissional está inserido, pode e indica o potencial da supervisão. Segundo o autor, através da análise do contexto, o profissional será capaz de identificar e intervir nos pontos cruciais de seu âmbito de trabalho, assim promovendo a melhora na qualidade assistencial, integrando os processos de cuidar e administrar, além de avaliar resultados com habilidade para empreender e realizar ações inovadoras. Neste sentido, o autor enfatiza o quanto é necessário que a todo tempo ocorra a busca de novas estratégias durante todo processo de supervisão.

Reafirmando esta ideia, os autores Vergílio, Toledo e Silva (2018), relatam que existem diversos modelos de instrumentos que já são utilizados na supervisão de enfermagem. No entanto, abordam que estes modelos estão interligados a dinâmica e organização de cada instituição, ou seja, segundo eles, cada supervisor poderá adaptar ou desenvolver da melhor forma possível um instrumento de avaliação, e isto dependerá apenas da demanda dos trabalhadores e pacientes que o local possui. 
Dessa forma, é possível perceber que não existe um instrumento criado especificamente para a avaliação, assim, permitindo que a instituição possa explorar ao máximo as ideias do supervisor, proporcionando planejamento, organização, qualificação e união entre a equipe.

Estes mesmos autores relatam que uma das formas de estratégias é justamente a realização de oficinas. A qual, irá possibilitar ao gestor desenvolver em sua equipe consciência/pensamento crítico sobre processos internos omitidos pela comunicação bloqueada, e atravessada por relações de poder institucionalizadas. Segundo eles, estas oficinas proporcionam entre o supervisor e a equipe, um momento o qual poderão falar sobre a sobrecarga, estresse, sentimentos, planejamento e a perspectiva em relação ao trabalho.

Neste mesmo sentido, os autores Gonge e Buus (2015), exemplificam outro tipo de estratégia, que seria justamente a oferta de novos cursos. Para eles, os profissionais, principalmente os de saúde, devem a todo tempo buscar novos níveis de evidência sobre sua área de conhecimento, assim a oferta de novos cursos, possuem como objetivo proporcionar treinamentos sobre supervisão clínica, a fim de preparar a todos para situações gerenciais. Os autores concluem que esta estratégia tem como objetivo tornar a assistência planejada e organizada.

Seguindo essa perspectiva educacional, os autores Oroná e Perciante (2020) descrevem sobre a identificação de um problema em uma unidade hospitalar referente a materiais e insumos, percebida pelo departamento de Administração de Enfermagem e pela própria comunidade de enfermagem. É interessante perceber que ao identificar o problema, é levantada a importância da gestão e supervisão atuando na formação permanente, e para tal, para facilitar e resolver o problema os autores aprimoraram um sistema já existente e criaram um curso.

Neste contexto, os autores trazem uma forma de instrumento de supervisão, que é o curso associado ao sistema, e relatam que ele permite um alcance mais efetivo e com resultados eficientes na saúde. Esse ponto de investimento na educação permanente é fator crucial dentro das categorias da supervisão. Pois, entende-se que o líder, ao exercer suas funções de conduzir e orientar sua equipe consegue identificar as falhas e problemas dos colaboradores e da assistência. Assim, com a identificação, o supervisor tende a resolver as falhas mediante a aplicação de meios/instrumentos que solucione e aprimore o trabalho.

Explorando mais a literatura, o estudo Husebo e Olsen (2019), apresentou uma análise sobre as atividades desempenhadas após a participação dos líderes (enfermeiros e médicos) de um curso de liderança dentro de um departamento de emergência. Mediante a essa metodologia, para a enfermagem, o curso trouxe a descrição de trabalhar confiança, qualidade, capacidade de resposta e eficiência quanto lideres diante das atividades desempenhadas em sua equipe.

Neste ponto, os autores supracitados, evidenciam como um curso de liderança pode ajudar na organização do serviçoe tornar o profissional autônomo e ciente de suas atividades privativas, assim, apresentando-se como uma ferramenta de supervisão, visto que, o objetivo do estudo foi acompanhar o resultado na prática após teoria.

Seguido essa mesma categoria, mas, distanciando-se do mais convencional, os autores Barroso et al. (2020), abordam sobre o desenvolvimento de um modelo de supervisão direcionado para as especificidades do ambiente. Nesse sentido, os autores desenvolveram um modelo denominado SafeCare. Para eles, esse modelo irá buscar acompanhar as mudanças advindas da tecnologia e avanços na saúde, ao mesmo tempo em que consiga abranger a diversidade clínica encontrada na assistência. De forma geral, o autor traz que esse modelo se caracteriza como um identificador de indicador sensível a supervisão de enfermagem.

Segundo o estudo, o SafeCare irá basear-se nos eixos do contexto o qual está-se inserido, cuidados de enfermagem, desenvolvimento profissional e supervisão. Esses eixos descritos serão compostos por quatro etapas, sendo elas: diagnóstico da situação, identificação a necessidade em supervisão clínica, implementação do modelo SafeCare e avaliação dos 
resultados. Assim, é perceptível a necessidade de identificar as reais necessidades dentro do serviço, e a partir disso desenvolver instrumentos de forma especifica para cada realidade, pois, só dessa forma a supervisão irá ocorrer de modo mais eficiente e singular, garantindo qualidade ao serviço, satisfação ao profissional e segurança ao paciente.

\section{Considerações Finais}

Através desse estudo nota-se que o supervisonador sofreu alterações ao longo do tempo, devido evolução e inovações do processo de trabalho, antes o mesmo possuía um cunho fiscalizador e punitivo, o que acarretava supressão de autonomia e fragmentação do trabalho, hoje a supervisão deve ser inerente ao enfermeiro, e sua execução deve ter foco em uma dimensão educativa, em detrimento da punição. Tendo em vista a relação da supervisão com a qualidade assistencial, nota-se que a mesma é imprescindível na prestação de serviços de excelência, pois no modelo contemporâneo a supervisão oferece subsídios para organização e avaliação do trabalho e identifica fragilidades e potencialidades que podem ser sanadas com a educação permanente.

Sabendo que os impactos da supervisão produzem resultados positivos na qualidade assistencial, designadamente a gestão do processo de trabalho, e o desenvolvimento contínuo do supervisor e do supervisionado, o enfermeiro deve aperfeiçoar seus conhecimentos científicos acerca da temática, e buscar estratégias e instrumentos que otimizem sua atuação profissional nos estabelecimentos de saúde. Com o presente estudo, foi possível identificar nas literaturas as seguintes ferramentas e instrumentos que auxiliam no fazer supervisivo: dados estatísticos, relatórios de serviços, fichas dos usuários, manuais, rotinas, mapas, roteiros, indicadores de saúde, oficinas e cursos de aperfeiçoamento.

Portanto, a questão deste estudo é expressiva tanto para os profissionais atuantes no desenvolvimento de suas práticas rotineiras, quanto para os acadêmicos de enfermagem, visto que esta é uma exigência do atual mercado de trabalho. Apesar da relevância do tema, notou-se que o mesmo deve ser mais discutido entre os estabelecimentos assistenciais de saúde e instituições de ensino superior, numa concepção articulada, que permita a tomada de decisão baseada em evidências.

\section{Referências}

Barroso, C., Carvalho, A. L., Augusto, C., Teixeira, L. O., Teixeira, A., \& Rocha, I. (2020). Implementação de um modelo de supervisão clínica em enfermagem-modelo SAFECARE. Revista ROL de Enfermería, 43(1), 50-58.

Buus, N., Delgado, C., Traynor, M., \& Gonge, H. (2018). Resistance to group clinical supervision: A semistructured interview study of non-participating mental health nursing staff members. International journal of mental health nursing, 27(2), 783-793.

Carvalho, N. A., Gama, B. M. B. M. \& Salimena, A. M. O. (2017). A supervisão sob a ótica dos enfermeiros: reflexos na assistência e trabalho em equipe. Revista de Administração em Saúde, 17(69).

Cecilio, H. P. M.; \& Oliveira, D.C. (2017). Modelos de revisão integrativa: discussão na pesquisa em Enfermagem. Congresso Ibero-Americano em InvestigaçãoQualitativa (CIAIQ), 2.

Chaves, L. D. P., Mininel, V. A., Silva, J. A. M. D., Alves, L. R., Silva, M. F. D., \& Camelo, S. H. H. (2017). Nursingsupervision for carecomprehensiveness. Revista brasileira de enfermagem, 70, 1106-1111.

Cofen (2018). Parecer $n^{\circ}$ 02, de 23 de março de 2018. Dispõe sobre a Organização de Enfermagem e definição da supervisão de enfermagem. http://www.cofen.gov.br/parecer-n-02-2018-cofen-ctln_61504.html

Coren (2015). Parecer $n^{\circ}$ 17, de 24 de abril de 2015. Dispõe sobre técnico e estagiário de enfermagem trabalhando sem a supervisão do enfermeiro. http://se.corens.portalcofen.gov.br/wp-content/uploads/2015/04/Parecer-Tecnico-17.2015.pdf

Dias, A. K. G., Toledo, L. V., Amaro, M. D. O. F., \& Siman, A. G. (2017). A percepção dos enfermeiros em relação ao seu papel gerencial no âmbito hospitalar. Rev. enferm. UFPE online, 2185-2194.

Dias, C. A., Santos, D. C., de Oliveira Matias, L., Servo, M. L. S., de Santana, C. L. A., \& Tanaka, L. H. (2018). Representa ções de supervisão na perspectiva dos enfermeiros coordenadores de um hospital de ensino. Revista Baiana de Enfermagem 32. 
Research, Society and Development, v. 10, n. 10, e465101018875, 2021 (CC BY 4.0) | ISSN 2525-3409 | DOI: http://dx.doi.org/10.33448/rsd-v10i10.18875

Ferreira, V. H. S., Teixeira, V. M., Giacomini, M. A., Alves, L. R., Gleriano, J. S., \& Chaves, L. D. P. (2019). Contribuições e desafios do gerenciamento de enfermagem hospitalar: evidências científicas. Revista Gaúcha de Enfermagem, 40.

Forte, E. C. N., Pires, D. E. P. D., Martins, M. M. F. P. D. S., Padilha, M. I. C. D. S., Ghizoni Schneider, D., \& Trindade, L. D. L. (2019). Processo detrabalho: fundamentação para compreender os erros de enfermagem. Revista da Escola de Enfermagem da USP, 53.

Galvão, M. C. B., \& Ricarte, I. L. M. (2019). Revisão sistemática da literatura: conceituação, produção e publicação. Logeion: Filosofia da informação, 6(1), 57-73.

Góis, R. M. O., \& Servo, M. L. S. (2017). Representação social da enfermeira sobre o processo de trabalho de supervisão hospitalar. In Congresso Internacional de Enfermagem (Vol. 1, No. 1).

Gonge, H., \& Buus, N. (2016). Exploring organizational barriers to strengthening clinical supervision of psychiatric nursing staff: a longitudinal controlled intervention study. Issues in mental health nursing, 37(5), 332-343.

Gonge, H., \& Buus, N. (2015). Is it possible to strengthen psychiatric nursing staff's clinical supervision? RCT of a meta-supervision intervention. Journal of Advanced Nursing, 71(4), 909-921.

Husebo, S. E., \& Olsen, O. E. (2019). Actual clinical leadership: a shadowing study of charge nurses and doctors on-call in the emergency department. Scandinavian journal of trauma, resuscitation and emergency medicine, 27(1), 1-9.

Johansson, D. (2015). Nursing clinical supervision project in a Neonatal Intensive Care and a Special Care Baby Unit: a best practice implementation project. JBI EvidenceSynthesis, 13(3), 247-257.

Kahl, C., Meirelles, B. H. S, Lanzoni, G. M. M, Koerich, C. \& Cunha, K. S. (2018). Ações e interações na prática clínica do enfermeiro na atenção primária à saúde. Revista da Escola de Enfermagem da USP, 52, 1-7.

Mendes, K. D. S., Silveira, R. C. D. C. P., \& Galvão, C. M. (2019). Uso de gerenciador de referências bibliográficas na seleção dos estudos primários em revisão integrativa. Texto \& Contexto-Enfermagem, 28.

Ministério da Saúde (BR) (2018). Resolução No 573, de 31 de janeiro de 2018. Recomendações do Conselho Nacional de Saúde à proposta de Diretrizes Curriculares Nacionais (DCN) do curso de graduação Bacharelado em Enfermagem. Diário Oficial da União, 213(1), 38.

Nasurdin, A. M., Tan, C. L., \& Khan, S. N. (2020). The Impact of Social Support on Nurses' Motivation: A Cross-Sectional Study in Malaysia. Asia Pacific Journal of Public Health, 32(8), 430-435.

Oroná, S. E. O., \& Perciante, M. I U. (2020). Gestión de recursos materiales "Guía para laaproximación diagnóstica de una unidadhospitalaria". Rev. urug. enferm; 15 (1).

Pinto, D. J. E., Santos, M. R., \& Pires, R. (2017). Relevance of indicators of clinical supervision strategies in nursing. Revista da Rede de Enfermagem do Nordeste, $18(1), 19$ 